\title{
Fixation Induced Blindness
}

\author{
Ahmad Yousef ${ }^{1}$ \\ ${ }^{1}$ School of Computational Science and Engineering, McMaster University, Hamilton, Ontario, Canada \\ *Correspondence: mohamas2@mcmaster.ca
}

\begin{abstract}
This article reports how fixation could convey visual stimuli to the invisibility region whether the stimuli are presented centrally or peripherally regardless the textures of the background. It also reports the impossibility of conveying visual stimulus to the invisibility region when the stimulus is not fixated, namely, when the stimulus is in motion.

We started in discussing how visual fixation could convey a centrally presented stimulus (pink horse) into the invisibility region under certain conditions, and why breaking the aforementioned invisibility by an intentional saccade away from the fixational point allows the stimulus to exert a ghostly horse but with complementary colours. Scientists had been hypothesizing that image aftereffect is caused by neural adaptation. In another word, the retinal photoreceptors \& its corresponding neurological pathways to the visual awareness might be being idle, namely, the visual respective field might be idle. Idle visual receptive field seems to be the best
\end{abstract} explanation of the present illusion, namely, we see the light grayish background turned to greenish in the aforementioned desensitized receptive field. Important to mention, fixation greatly inhibits the spontaneous saccadic eye movements, and thus, it reduces the rooms of the receptive field remapping. Namely, every visual space will be possibly have unchangeable visual map in the brain. To arrest the aforementioned statements, we built a running stimulus to disallow the overlapping of the image and its aftereffect, and we found that the image cannot disappear. In another word, the visual awareness of the aforementioned stimulus would have ghostly \& cloudy green balls in between the original materials (purple balls). The previously mentioned finding confirms the role of the spontaneous saccadic movement in promoting visibility \& preventing the blindness, also see reference 1 . We ended this research with asserting whether the claims against Emmert's law which raised doubts about the accurate compliance of the aforementioned law and sizedistance invariance hypothesis. Weirdly enough, the claims are correct as if the image aftereffect projected against distant wall is following the dynamical visual angle but not the static one.
Introduction \& preliminary findings

Contrast sensitivity function describes the visible region that can trigger visual awareness produced by the human brains in gray scale, see reference 2 . Colour, however, is an additional parameter that eventually generate the function of color visibility, see reference 3 , and 4 . When the aforementioned functions are properly integrated, the visibility region of human visual awareness can be described. In this article, we deeply study multiple factors in Troxler effect, see reference 5. The aforementioned effect allows visual fixation to terminate the visual awareness of the stimulus; possibly because of the inhibition of the spontaneous saccadic eye movements caused by the fixation. The aforementioned sensitivity functions might be therefore insufficient to explain how fixation could induce blindness, namely, because these functions are obtained under normal visual conditions, including but not limited to, the spontaneous saccadic eye movements. Further analytical depth are therefore required to understand the neuro-machinery of the present illusion, namely, a novel contrast sensitivity function should be constructed with fulsome consideration of visual fixation. Important to mention, physiologists had been commonly agreed that the retinal layer is mapped in the brain, namely, there are neurological road from each photoreceptor to designated brain areas. We think that in the end of these neurological roads the visual awareness emerges, see reference 6 . Interesting to say, idle neurological roads could also convey the conscious information, namely, when the neurological roads to the awareness of the pink horse are desensitized, the visual awareness kept the information but with few modifications, namely, inverted colors. Consistently, Hsieh and Tse showed that the induced blindness by fixation mostly occurred in the brain, but not in the eyes, see reference 7. Speaking in cellular terms, it is believed that trichromacy occurs at the photoreceptors' sensing stage, see reference 8; but the opponent processes are expressed at the retinal ganglion cells' stage and in several parts of the brain, including but not limited to, the neocortex, see reference 9. In opponent process theory, 
the system works based on the opposing color effect of red-green, blue-yellow, and light-dark; however, it is the visual neural processes of various types of photoreceptors that are opposed. Since Hering's theory is a neuropsychological structure that describes a wide spectrum of behavioral dynamics, including color vision; it therefore might be therefore able to give another explanation to the present illusion. Important to mention, there are two potential mechanisms for color constancy; the unconscious inference, see reference 10; and the sensory adaptation, see references 11 and 12. Physiologists suggested 'color constancy' relates to variations in retinal cells and visual cortical areas, see references, 13, 14, and 15. The present illusion, however, seems to follow the sensory adaptation theory, namely because the illusory image aftereffect is mostly triggered by idle neurological pathways to the visual awareness. Important to emphasize, the first experiment consisted of pink horse that's continuously visible to the visual awareness; however, prolonged fixation at a precise point convey the horse to the 'invisible' region, namely the horse disappears from the visual awareness, see reference 16. As previously discussed, any intentional or directional saccade after the blindness induced by fixation will reveal the secrets of this invisibility, namely, a greenish ghost horse will emerge in the direction of the performed saccade; the intentional saccade that should terminate the invisibility. The magenta horse will, afterwards, return to its normal state, but with ghostly greenish clouds around the horse. The aforementioned invisibility and then detachment of the two horses are outstanding observation, it theorizes that the importance of the spontaneous saccadic eye movements in human vision. The second experiment strongly agrees with the aforementioned theorization despite the stimulus is presented in the peripheries, namely, the stimulus can be conveyed to the visual invisibility region if it is stationary! If the stimulus is in motion, however, ghostly \& cloudy green balls will appear in between the actual stimuli, the purple balls; evidencing that the idle neurological roads to the visual awareness may still carry the information (the inverted colored horse). The third experiment, however, confirms the recent claims of inaccuracy of Emmert's law, and it also raises a few doubts about whether 'the idle neurological roads to the visual awareness' is the only contributor of the projected aftereffect image.

\section{Materials \& Methods}

Twelve human subjects, with dilated pupils, were recruited to participate in this study. The first experiment consisted of one horse centrally presented but with three different textures; namely, each human subject has to be tested for three times with three different textures of backgrounds to ensure not only data finetuning but the contribution of texture perception on this color illusion. The horse subtends maximally 13 degrees visual angles for both length and width. The stimulus is lightly blurred to trigger Troxler's fading. The subjects are requested to perform up-right saccade after experiencing the invisibility, and to describe what they had seen at the corresponding visual space. The second experiment consisted of 12 purple balls distributed in a circular shape, each of these balls subtends 2.43 degrees visual angle. The aforementioned experiment consisted of two condition, stationary versus motion with rotational frequency of $0.25 \mathrm{~Hz}$. Finally, the third experiment is about repeating the first experiment but after the invisibility's experience, the subject to execute a saccade toward a fixational point print in a distant wall, namely, $310 \mathrm{~cm}$ away from the subjects. Five squares are drawn around the fixational point in the wall, namely, the sizes of the squares $1.2,1$, $0.8,0.6$, and 0.4 times of the actual size of the horse. Because the wall is very far from the subjects, the horse should subtend $71 \mathrm{~cm}^{2}$ to have an equivalent visual angle of the horse viewed in the computer monitor. The behavioral data were collected manually, and then averaged among the human subjects to estimate the simplest statistical values, namely, the average and the standard error of the mean of the visibility strength compared to the reference.

\section{Results and Conclusion}

Concerning the first experiment, as shown in the upcentral infographic, visual fixation drastically diminishes the visibility of the horse. Through their precise fixation, the twelve human subjects were able to make the horse mostly invisible from their visual awareness with almost no noticeable contribution of the texture perception on the present color illusion. The performed saccade after the invisibility enable the human subjects to see the greenish 'ghostly' horse. Regarding the second experiment, however, all of the human subjects were able to 'completely' convey to the purple balls to the invisible region, however, none of them were able to do that when the stimulus is in motion, namely, their report visual 
awareness was as follow: ghostly \& cloudy green balls in the intermediate areas between the purple balls (the real stimulus). The aforementioned experiment strongly agrees with the general hypothesis describes in the abstract, namely, the illusion might be explained by stating the following: There are desensitized receptive fields that allows all of the colors but not the magenta to pass. It also evidences that the idle neurological roads to the visual awareness may still carry the information (the inverted colored horse).

We finalized the present study by asserting whether the claims against Emmert's law which raised doubts about the accurate compliance of the aforementioned law and sizedistance invariance hypothesis, see reference 17. Important to remind, it was found that that near-vision tends to have dilated pupil \& rounded lens, but far-vision tends to have constricted pupil \& flatter lens, see reference 18. Interestingly enough, the claims are accurate as if the image aftereffect projected against distant wall is following the dynamical visual angle, see reference 19 . Namely, when the human subjects project the aftereffect image against the a distant wall \& they fixated at the fixational point, all of them had noticed a noticeable shrinkage against the actual size based on the visual angle calculations with the following values; mean size $=0.84 \&$ $S E M=0.3$. Important to emphasize, according to Emmert's law, the projected image must be way greater than the image in the computer monitor, and it should follow the geometrical calculations of the visual angle. In this study, however, precise compliance is valid; this might be because when the human subjects fixate their attention against a distant wall, their pupils might be slightly constricted, and therefore the aftereffect images appear to be smaller. Interesting to mention, the neural activities in the primary visual reflects the perceived size but not retinal of the aftereffect images, see reference 20 . The aforementioned extraordinary finding might raise several speculative questions; whether the brain itself emits personalized physical particles (tachyonic emission) in the extra physical dimensions, see reference 6; otherwise, what's purpose of the changes of pupil-lens dynamics if everything are exclusive to the brain neurophysiological processes? Additionally, why V1 has greater neural activities regardless the retinal size of afterimage?

Essential Notifications Interesting to mention, Kitaoka strawberries are dark gray but they are perceived as red when the image peripheral areas are cyan, see reference 21 ; as if the desensitized retinal peripheries generate ghostly clouds of opposite color during the spontaneous saccades, and therefore the strawberries appears in red color. To comply to the ethics of scientific research, and to the declaration of Helsinki; readers should have direct access to the stimuli, but other martials are available upon request, see reference 16 .
Advisably, download the videos for the best quality of experience. Wait for a few seconds to allow adaptation to the pupil and the lens; the illusion afterwards should work. For rapid validation process, watch the stimuli in moderate brightness with dilated pupils. Lastly, we wish to collaborate with scholars have access to professional eye tracker, and brain imaging

facilitations in challenging investigations, namely, to achieve critical analyses of spatiotemporal properties of the theorical two distinct V4 areas (fovea versus peripheries), and other neocortical and subcortical areas to comprehend the neurophysiological reasons of the FIB. Enquiries should be sent to the author.

Transactional References

[1] Martinez-Conde, etal. (2004). The role of fixational eye movements in visual perception. Nature Reviews Neuroscience. [2] Campbell, and Robson (1968) Application of Fourier analysis to the visibility of gratings. J Physiology.

[3] Jean-Pierre Menu (1984). Effects of Colour on the Contrast Sensitivity Function as a Function of Eccentricity. Internat. Visual Field Symposium.

[4] K. Mullen (1985). The contrast sensitivity of human colour vision to red-green and blue-yellow chromatic gratings. Journal of Physiology.

[5] Troxler, (1804) "Über das Verschwinden gegebener Gegenstände innerhalb unseres Gesichtskreises". Ophthalmologische Bibliothek.

[6] Yousef, Ahmad. 2019. "Consciousness Might Be Localized in Extra Physical Dimensions." PsyArXiv. doi:10.31234/osf.io/angc8.

[7] Hsieh; Tse (2006). "Illusory color mixing upon perceptual fading and filling-in does not result in 'forbidden colors'". Vision Research.

[8] Thomas Young, (1802). Bakerian Lecture: On the Theory of Light and Colours. Phil. Trans. R. Soc. Lond. 92:12-48. doi: 10.1098/rstl.

1802.0004 .

[9] Ewald Hering, (1878) "Opponent-process Theory." Expanded by Solomon.

[10] Judd (1940). Hue saturation and lightness of surface colors with chromatic illumination. Journal of the Optical Society.

[11] Helson (1943). Some factors and implications of color constancy. Journal of the Optical Society of America.

[12] Ewald Hering (1920). Grundzüge der Lehre vom Lichtsinn. Berlin; Springer.

[13] Semir Zeki (1980). The representation of colours in the cerebral cortex. Nature.

[14] Semir Zeki (1983). Colour coding in the cerebral cortex: The reaction of cells in monkey visual cortex to wavelengths and colours. Neuroscience.

[15] Hood (1998). Lower-Level Visual Processing and Models of Light Adaptation. Annual Review of Psychology.

[16] You may find the stimuli in the following link:

https://drive.google.com/drive/folders/1-

jp5A4fjbVPobO2aGIUBGzw3LQfzKyOb

[17] Lou (2007). Apparent afterimage size, Emmert's law, and oculomotor adjustment. Perception.

[18] Yousef, A. 2019. "Linking Dynamics Between Pupil and Lens." engrXiv. doi:10.31224/osf.io/9c3vk.

[19] Yousef, Ahmad. 2019. "The Dynamical Visual Angle." PsyArXiv. doi:10.31234/osf.io/f2kr6.

[20] Sperandio, etal. (2012). "Retinotopic activity in V1 reflects the perceived and not the retinal size of an afterimage". Nat. Neurosci. [21] Baysan, \& Macpherson (2017) "Grey strawberries" in: The Illusions Index. 